\title{
Complications and Risk Factors of Patients Undergoing Computed Tomography-Guided Core Needle Lung Biopsy: A Single-Center Experience
}

\author{
Waseem M. Hajjar ${ }^{1}$, Ibrahim M. Fetyani ${ }^{2}$, Rayan M. Alqarni ${ }^{2}$, Fahad A. Alajlan ${ }^{2}$, Fouad F. Bahgat ${ }^{2}$,
} Sultan R. Alharbi ${ }^{3}$

1. Surgery, College of Medicine, King Saud University, Riyadh, SAU 2. General Surgery, King Saud University, Riyadh, SAU 3. Interventional Radiology, King Saud University, Riyadh, SAU

Corresponding author: Waseem M. Hajjar, whajjar@ksu.edu.sa

\section{Abstract \\ Objectives}

To determine the risk factors and complications of transthoracic computed tomography (CT)-guided core needle lung biopsy.

\section{Methods}

This is a retrospective study of 124 patients who underwent CT-guided core lung biopsy in King Khalid University Hospital (KKUH), Riyadh. This retrospective study was conducted between January 2016 and January 2020. Patient data were collected using a standardized data form that was entered into an Excel sheet in accordance with the variables. The Statistical Package for the Social Sciences software (SPSS, version 24.0 [SPSS Inc., Chicago, IL, USA]) was used to compute for the risk of complications after CT-guided core lung biopsy and perform all statistical comparisons, and the results were reported.

\section{Results}

The overall complication rate due to CT-guided core needle biopsy was $34.7 \%(43)(\mathrm{P}<0.001)$ of the total sample. Of the total complications, 69.76\% $(n=30)$ had pneumothorax, 20.94\% $(n=9)$ had hemorrhage, 6.98\% $(n=3)$ had both pneumothorax and hemorrhage, and 2.32\% $(n=1)$ had both air embolism and pneumothorax. Of all patients who developed pneumothorax, $20 \%(n=6)$ required chest tube insertion. Patients with secondary chronic obstructive pulmonary disease (COPD) had a complication rate of $80 \%$ among the whole sample. Lung lesions less than $3 \mathrm{~cm}$ had a complication rate of $48.8 \%(\mathrm{P}<0.034)$. The needle size showed a higher rate of complications between 20 and 18 gauge with $47.4 \%(n=9)$ and $32.4 \%(n=$ 34), respectively.

\section{Conclusions}

Review began 06/21/2021 Review ended 07/23/2021 Published 08/05/2021

\section{() Copyright 2021}

Hajjar et al. This is an open access article distributed under the terms of the Creative Commons Attribution License CC-BY 4.0., which permits unrestricted use, distribution, and reproduction in any medium, provided the original author and source are credited.
We conclude that CT-guided lung biopsy is a well-established low-risk procedure that is less invasive. However, it still carries a risk of complications with some risk factors, such as small lung lesion size and secondary COPD.

\author{
Categories: Cardiac/Thoracic/Vascular Surgery, Radiology \\ Keywords: ct-guided core needle biopsy, risk factors, complications, pneumothorax, haemorrhage
}

\section{Introduction}

In modern medicine, computed tomography (CT) of the lung is commonly used to screen for primary lung cancer or metastatic cancer, leading to the discovery of more patients with lung masses [1-3]. A minimally invasive procedure such as core needle biopsy reduces the need for open surgical lung biopsy. Such procedures help confirm the diagnosis of suspected lung lesions in addition to several screening programs, one of which is the screening of high-risk groups for lung cancer. It has become widely popular across various institutions and is in constant demand for the diagnosis of different lung diseases [4,5].

Nevertheless, a core needle biopsy is associated with several complications, mainly pneumothorax and hemothorax and their corresponding risk factors [6]. Risk factors include lesion size, depth, the presence of primary emphysema, age, sex, location, shape, the presence of atelectasis, fissure in the needle tract, needle entry angle, number of pleural punctures, the experience of the operator, and procedure duration. Many previous studies have reported on the complications and risks of this procedure, but few have shown the correlation of these factors with the diagnostic yield of different needle gauge sizes used $[1,2,7]$.

Complication rates have varied across different studies, ranging from $8 \%$ to $69 \%$ [1,2]. Pneumothorax is the 
most common complication following core needle biopsy [8]. Pneumothorax is defined as the presence of air in the pleural space. This has many causes mainly iatrogenic, especially in CT-guided core needle biopsy.

Most pneumothoraces are treated conservatively without chest tube placement and are considered minimal. However, some cases require further attention and subsequent placement of a chest tube [6].

A study reported that the incidence of pneumothorax was $15.4 \%$ in patients undergoing the procedure and chest tube placement was needed in only $22.8 \%$ of all pneumothoraces [1]. The position of the patient, length of the needle, and history of smoking were the most important risk factors for developing pneumothorax $[1,4,8-10]$.

In some studies, pulmonary hemorrhage, including hemoptysis, intraparenchymal hemorrhage, and hematoma were the most common complications encountered, with an incidence rate of $45.4 \%$ in one study [9]. The correlation between this complication and several risk factors was analyzed, maximal diameter and needle length were the most significant risk factors [10]. Some controversy remains when comparing coreneedle biopsy to fine-needle aspiration as some studies have reported a higher complication rate in core needle biopsy than in fine-needle aspiration [4]. However, other studies have found that both core needle biopsy and fine-needle aspiration have the same complication rate and suggested the use of the former as it has a better diagnostic outcome [11].

Many studies have shown that CT-guided core needle biopsy is a reliable diagnostic procedure with a low incidence of complications, and it is used for thoracentesis and other therapeutic lung procedures [12]. CTguided lung biopsy is considered the modality of choice because of its reliability and low complication rate. Usually, biopsy specimens are categorized as malignant, benign, or undetermined. Several factors play a role in the integrity and adequacy of specimens; however, a CT-guided core needle biopsy is known to produce a high diagnostic yield [13-15].

\section{Materials And Methods}

\section{Patients}

The present study was approved by the institutional review board (IRB) committee of King Saud University. The study was conducted in accordance with international ethical standards. Patient names and other confidential information were not used in this study; therefore, patient consent was waived.

The study was carried out in Riyadh, Saudi Arabia, as a single-center experience in a tertiary university hospital. Data from all patients collected from the Interventional Radiology Department, including that from all patients who underwent CT-guided core lung biopsy from January 2016 to January 2020, were included in this study.

\section{CT-guided core needle lung biopsy technique}

All patients underwent CT-guided needle core lung biopsy by a consultant interventional radiologist. After reviewing previous imaging and obtaining informed consent, the patient was placed in the most suitable position (prone, lateral, or supine) on the CT scan table. Under sterile conditions, local anesthesia, and conscious sedation, lung core biopsies were performed using an $18 \mathrm{G}$ or $20 \mathrm{G}$ coaxial quick core biopsy needle set (Cook, Bloomington, IN, USA) (Figure 1). The needle set size was determined based on the size of the lesion, background lung disease, performance preference, and item availability. Generally, a 20G needle is used for smaller lesions, chronic obstructive pulmonary disease (COPD) patients, and difficult mass locations to reduce the risk of complications. Post-biopsy CT chest scanning was performed at the end of the procedure to determine if there was a complication. Subsequently, the patient was moved to the surgical ward for observation for 3-4 h. Before discharging the patient, a chest x-ray was also performed to exclude late complications, mainly pneumothorax. 


\section{Cureus}

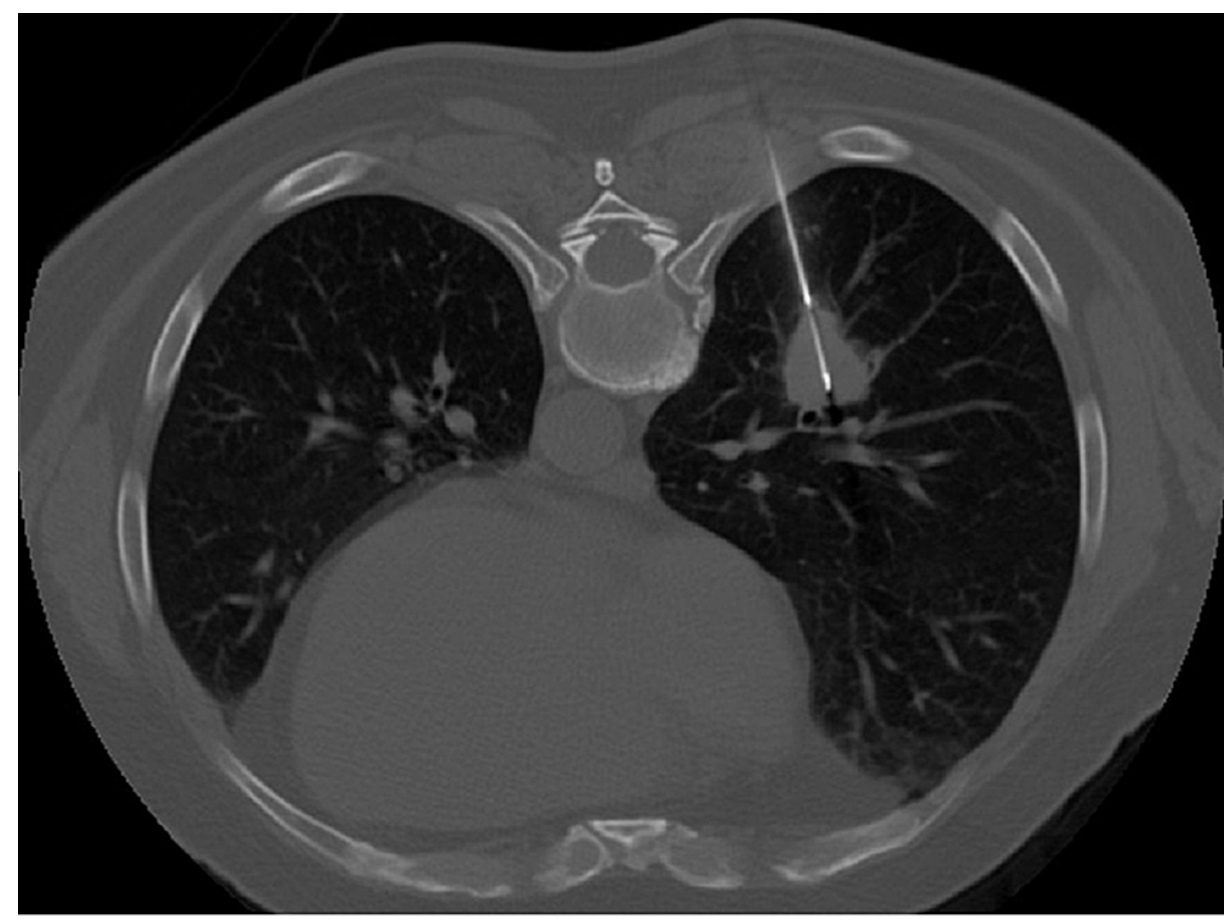

FIGURE 1: CT-guided lung biopsy of a right lung lesion (prone position).

CT - computed tomography

\section{Data collection}

This study was conducted as a retrospective study that included 124 patients who underwent CT-guided core lung biopsy. Patient data were collected using a standardized data form. The data were entered into an Excel sheet, and missing data were considered not present. All CT-guided needle biopsies conducted during the period mentioned were reviewed. The outcomes and complications of all the procedures were documented and analyzed.

Inclusion criteria included all patients who underwent CT-guided core needle lung biopsy using an 18G needle or 20G needle in the period mentioned. The exclusion criteria included insufficient data, repetitive data, and canceled procedures. The initial sample size was 301 patients, but this was reduced to 124 patients.

The variables included patients' demographic data, characteristics, lesion variables, and procedure-related variables. Data were obtained from electronic patient files (e-SiHi) and the Picture Archiving and Communication System (PACS). All CT-guided core needle biopsies conducted during the study period were collected and analyzed, and all data were organized in an Excel sheet and subsequently analyzed using Statistical Package for the Social Sciences (SPSS), and the outcomes were reported. All the mentioned variables and outcomes included were obtained from similar studies that were studied and mentioned in the literature [1-3].

\section{Data and statistical analysis}

The risk of complications after CT-guided core needle biopsy and all statistical comparisons were studied using. Statistical analysis was performed using the SPSS version 24.0 software (SPSS Inc., Chicago, IL, USA). Descriptive statistics were reported as mean \pm standard deviation for continuous variables and counted as a percentage for categorical values. A total of 124 patients were categorized into two groups based on which needle gauge was used (18G vs. 20G), and then divided into two subgroups depending on the presence of complications. The variables were compared using the chi-square test, Fisher's exact test, binomial test, and Mann-Whitney U test. The Student's t-test was used for two independent groups. Statistical significance was set at $\mathrm{P}<0.05$.

\section{Results}

This study correlated with different variables regarding the presence of complications. The number of cases that developed procedure-related complications had a prevalence of $34.7 \%(n=43)$, with a significant Pvalue of 0.001 . Demographic characteristics, including age, sex, and smoking, showed no significant correlation with complications. Disease-related risk factors namely location and presence of primary 


\section{Cureus}

emphysema showed no correlation as well. Meanwhile, lung lesion size showed a significantly high incidence of complications in those with less than $3 \mathrm{~cm}$ with a p-value of 0.034 . Procedure-related risk factors, including patient position, lesion depth, and the number of pleural punctures, showed no statistical significance with respect to the incidence of complications (Table 1). Of the total sample, the incidence of complications and pneumothorax was $24.2 \%(n=30)$, where $7.3 \%(n=9)$ had hemorrhage, $0.8 \%(n=1)$ had both air embolism and pneumothorax, and 2.4\% $(n=3)$ had both pneumothorax and hemorrhage. The total prevalence of different complications demonstrated a significant $\mathrm{P}$-value of $<0.0001$ (Figure 2 ). The complications were then correlated with the needle gauge used. A significant difference was found for hemorrhage, which occurred in 5.7\% ( $n=6)$ and $15.8 \%(n=3)$ when $18 \mathrm{G}$ and $20 \mathrm{G}$ were used, respectively, with a P-value of 0.141 . Pneumothorax occurred in $23.8 \%(n=25)$ of patients when $18 \mathrm{G}$ was used, compared to $26.3 \%(n=5)$ when $20 \mathrm{G}$ was used. Additionally, pneumothorax associated with hemorrhage occurred simultaneously in $1.9 \%(\mathrm{n}=2)$ of patients when $18 \mathrm{G}$ was used and $5.3 \%(\mathrm{n}=1)$ when $20 \mathrm{G}$ was used.

Meanwhile, no complications were seen in 67.6\% (71) and 52.6\% (10) of patients when 18G and 20G needles were used, respectively (Table 2). There was a single case of an air embolism associated with pneumothorax when an $18 \mathrm{G}$ needle was used. 


\section{Cureus}

\begin{tabular}{|c|c|c|c|c|c|c|}
\hline \multirow{2}{*}{ Variables } & & \multicolumn{2}{|c|}{ Complication } & \multicolumn{2}{|c|}{ No complication } & \multirow{2}{*}{ P-value } \\
\hline & & $\mathrm{N}$ & $\%$ & $\mathrm{~N}$ & $\%$ & \\
\hline Number of cases & & 43 & (34.7 \%) & 81 & (65.3\%) & 0.001 \\
\hline \multirow{2}{*}{ Age } & $\geq 60 y$ & 20 & $(46.5 \%)$ & 47 & $(58 \%)$ & \multirow{2}{*}{0.221} \\
\hline & $<60 y$ & 23 & (53.5 \%) & 34 & (42.0 \%) & \\
\hline \multirow{2}{*}{ Gender } & Male & 32 & (74.4 \%) & 48 & (59.3 \%) & \multirow{2}{*}{0.093} \\
\hline & Female & 11 & $(25.6 \%)$ & 33 & (40.7\%) & \\
\hline \multirow{3}{*}{ Patient position } & Lateral & 13 & (30.2 \%) & 11 & (13.6 \%) & \multirow{3}{*}{0.064} \\
\hline & Prone & 17 & (39.5 \%) & 34 & (42.0\%) & \\
\hline & Supine & 13 & (30.2 \%) & 36 & (44.4%) & \\
\hline \multirow{2}{*}{ Smoking $\mathrm{Hx}$. } & Yes & 22 & (51.2 \%) & 29 & (35.8 \%) & \multirow{2}{*}{0.098} \\
\hline & No & 21 & (48.8 \%) & 52 & (64.2\%) & \\
\hline \multirow{2}{*}{ Lung lesion size (in cm) } & $\leq 3.0$ & 21 & (48.8 \%) & 24 & (29.6 \%) & \multirow{2}{*}{0.034} \\
\hline & $\geq 3.1$ & 22 & (51.2 \%) & 57 & (70.4\%) & \\
\hline \multirow{3}{*}{ Lesion depth (in cm) } & 0 & 13 & (30.2 \%) & 35 & (43.2\%) & \multirow{3}{*}{0.333} \\
\hline & $0.2-3$ & 25 & (58.1 \%) & 40 & (49.4 \%) & \\
\hline & $>3$ & 5 & (11.6 \%) & 6 & (7.4 \%) & \\
\hline \multirow{4}{*}{ Comorbidities } & None & 27 & (62.8 \%) & 53 & (65.4 \%) & \multirow{4}{*}{0.863} \\
\hline & Lung disease & 10 & (23.3 \%) & 14 & (17.3\%) & \\
\hline & Infections & 3 & $(7.0 \%)$ & 7 & (8.6 \%) & \\
\hline & Miscellaneous & 3 & $(7.0 \%)$ & 7 & $(8.6 \%)$ & \\
\hline \multirow{3}{*}{ Severity of the primary emphysema } & None & 40 & (93.0 \%) & 76 & (93.8 \%) & \multirow{3}{*}{0.400} \\
\hline & Moderate & 2 & $(4.7 \%)$ & 1 & $(1.2 \%)$ & \\
\hline & Severe & 1 & $(2.3 \%)$ & 4 & $(4.9 \%)$ & \\
\hline \multirow{2}{*}{ Number of pleural punctures } & Single & 34 & (79.1 \%) & 68 & $(84.0 \%)$ & \multirow{2}{*}{0.322} \\
\hline & Multiple & 9 & (20.9 \%) & 13 & $(16.0 \%)$ & \\
\hline \multirow{3}{*}{ Lesion location } & Right lung lesion & 22 & (51.2 \%) & 41 & $(50.8 \%)$ & \multirow{3}{*}{0.050} \\
\hline & Left lung lesion & 20 & $(46.5 \%)$ & 27 & (37.9\%) & \\
\hline & Mediastinal & 1 & $(2.3 \%)$ & 13 & (11.3\%) & \\
\hline
\end{tabular}

TABLE 1: Patient's biographic and lesion variables. 


\section{Cureus}

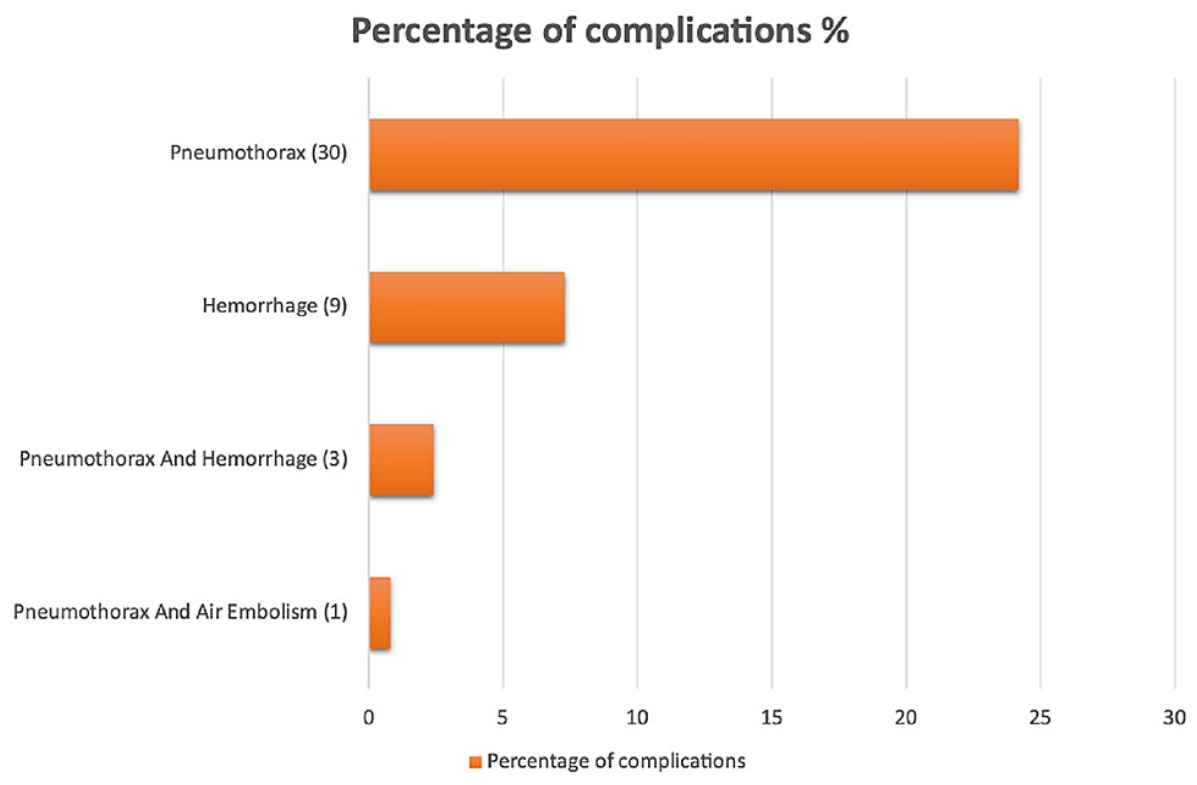

FIGURE 2: Total percentages of complications related to core needle biopsies.

\begin{tabular}{|c|c|c|c|c|c|}
\hline \multirow{3}{*}{ Complications } & \multicolumn{4}{|c|}{ Size of needle } & \multirow{3}{*}{ P-value } \\
\hline & \multicolumn{2}{|c|}{$18(n=105)$} & \multicolumn{2}{|c|}{$20(n=19)$} & \\
\hline & $\mathrm{N}$ & $\%$ & $\mathrm{~N}$ & $\%$ & \\
\hline Haemorrhage & 6 & $(5.7 \%)$ & 3 & $(15.8 \%)$ & 0.141 \\
\hline Pneumothorax & 25 & $(23.8 \%)$ & 5 & $(26.3 \%)$ & 0.508 \\
\hline No complication & 71 & $(67.6 \%)$ & 10 & (52.6 \%) & 0.207 \\
\hline Pneumothorax and Haemorrhage & 2 & $(1.9 \%)$ & 1 & $(5.3 \%)$ & 0.396 \\
\hline Pneumothorax and Air embolism & 1 & $(1.0 \%)$ & 0 & $(0 \%)$ & 0.847 \\
\hline
\end{tabular}

TABLE 2: Complications in correlation to needle size.

\section{Discussion}

CT-guided core needle biopsy has become a widely used tool and diagnostic method for the evaluation of pulmonary lesions. Pneumothorax and pulmonary hemorrhage are by far the most frequent complications. Other rare adverse events include hemorrhage, infection, needle tract seeding, and systemic air embolism [5]. The complication rates in this retrospective study were as follows: the total incidence of complications was $34.7 \%(n=43)$, pneumothorax had the highest incidence among all complications with $69.76 \%(n=30)$ of all patients that developed complications, while pneumothorax developed in only $20 \%(n=6)$, with a Pvalue of 0.001 with subsequent chest tube insertion. Additionally, hemorrhage occurred in $20.94 \%(n=9)$, pneumothorax associated with hemorrhage in $6.98 \%(n=3)$, and of those, only one patient needed a chest tube insertion, while air embolism associated with pneumothorax was found in only $2.32 \%(n=1)$. We found all complication rates to be consistent with those found in previously published papers $[1,4]$.

Of all risk factors that were studied, smaller lung lesion size and COPD patients had a higher incidence of complications. This correlation with smaller lesions could be explained by repeated attempts to acquire an adequate biopsy sample from the lesion, resulting in more damage to the lung parenchyma and increasing the chance of complications. Regarding the presence of complications in correlation to comorbidities, the incidence of complications in patients with comorbidities compared to healthy subjects was higher, with a prevalence of $37.2 \%$ and $34.6 \%$, respectively. Comorbidities were classified into multiple diagnoses, of which only secondary COPD was statistically significant, with a complication incidence of $80 \%$ of total secondary 
COPD patients. Patients with secondary COPD tend to have parenchymal disruption of varying degrees, which could explain the higher incidence of complications in these patients. Other correlated demographic lesion-related and procedure-related factors such as age, smoking, lesion depth, lesion location, and patient position did not show a clear association in our study. However, mixed results are found in the current literature; some studies did not show a correlation with any lesion characteristics, but these are the minority [11].

A variety of studies have compared core needle biopsy and fine-needle aspiration in terms of complications. One systematic review recommends the use of core needle biopsy over fine-needle aspiration because of the same rate of complications with a better diagnostic yield [11]. Additionally, one meta-analysis showed that core needle biopsy did not have significant risk factors for overall complications [4]. However, physicians tend to use a large needle size to obtain large histological samples, as well as to avoid the complications arising from smaller needle sizes, which led to the rarity of using 20G needle biopsies in our institution. Although the correlation between needle size and incidence of complications was not statistically significant in our study, there was a tendency towards a higher complication rate when using the $20 \mathrm{G}$ needle by $47.37 \%(\mathrm{n}=9)$. Nevertheless, $18 \mathrm{G}$ needles were associated with an incidence of complications of $32.38 \%(n=34)$, which may be due to the use of multiple attempts of needle insertion using the smaller gauge, and the sample size of the complications in the $18 \mathrm{G}$ group was significantly higher than that in the 20G group. Thus, this correlation needs more research to obtain a clearer approach.

The limitations of this research include a small sample size and a single-institution study, thus making several risk factors statistically insignificant. The inability to separate multiple co-occurring risk factors correlated individually with complication incidence.

\section{Conclusions}

We conclude that CT-guided lung biopsy is a well-established and low-risk procedure for the diagnosis of lung pathologies. It is also less invasive but associated with a risk of developing complications mainly pneumothorax and hemorrhage. However, small lung lesion size and secondary COPD, both carry a risk of developing complications related to the core needle biopsy, which could be explained by the repetitive attempts to obtain the sample from this small lesion, and also due to the parenchymal pathology in COPD patients.

We recommend further investigations in a multicenter study with a larger sample size to explore the different variables that were not statistically significant but trending toward significance.

\section{Additional Information \\ Disclosures}

Human subjects: Consent was obtained or waived by all participants in this study. Institutional Review Board (IRB) Committee of King Saud University issued approval No. E-19-4502. The study was conducted in accordance with international ethical standards. Patient names and other confidential information were not used in this study, and all unnecessary information was considered confidential. Animal subjects: All authors have confirmed that this study did not involve animal subjects or tissue. Conflicts of interest: In compliance with the ICMJE uniform disclosure form, all authors declare the following: Payment/services info: All authors have declared that no financial support was received from any organization for the submitted work. Financial relationships: All authors have declared that they have no financial relationships at present or within the previous three years with any organizations that might have an interest in the submitted work. Other relationships: All authors have declared that there are no other relationships or activities that could appear to have influenced the submitted work.

\section{Acknowledgements}

The authors would like to thank the Saudi Thoracic Society (STS) for their support.

\section{References}

1. Ozturk K, Soylu E, Gokalp G, Topal U: Risk factors of pneumothorax and chest tube placement after computed tomography-guided core needle biopsy of lung lesions: a single-centre experience with 822 biopsies. Pol J Radiol. 2018, 83:e407-14. 10.5114/pjr.2018.79205

2. Padrão E, Rodrigues M, Guimarães S, et al.: Diagnostic yield and complications of transthoracic computed tomography-guided biopsies. Respiration. 2018, 96:455-63. 10.1159/000489934

3. Lang D, Reinelt V, Horner A, Akbari K, Fellner F, Lichtenberger P, Lamprecht B: Complications of CT-guided transthoracic lung biopsy: a short report on current literature and a case of systemic air embolism. Wien Klin Wochenschr. 2018, 130:288-92. 10.1007/s00508-018-1317-0

4. Heerink WJ, de Bock GH, de Jonge GJ, Groen HJ, Vliegenthart R, Oudkerk M: Complication rates of CTguided transthoracic lung biopsy: meta-analysis. Eur Radiol. 2017, 27:138-48. 10.1007/s00330-016-4357-8

5. Jelitto-Gorska M, Studniarek M, Durawa A, Marjanski T, Szurowska E: Percutaneous lung needle biopsies utility and complications in various chest lesions: a single-institution experience. Pol J Radiol. 2018, 83:e103-8. 10.5114/pjr.2018.75825 
6. Wu CC, Maher MM, Shepard JA: Complications of CT-guided percutaneous needle biopsy of the chest: prevention and management. AJR Am J Roentgenol. 2011, 196:W678-82. 10.2214/AJR.10.4659

7. Boskovic T, Stanic J, Pena-Karan S, et al.: Pneumothorax after transthoracic needle biopsy of lung lesions under CT guidance. J Thorac Dis. 2014, 6 Suppl 1:S99-107. 10.3978/j.issn.2072-1439.2013.12.08

8. Hiraki T, Mimura H, Gobara H, Shibamoto K, Inoue D, Matsui Y, Kanazawa S: Incidence of and risk factors for pneumothorax and chest tube placement after CT fluoroscopy-guided percutaneous lung biopsy: retrospective analysis of the procedures conducted over a 9-year period. AJR Am J Roentgenol. 2010, 194:809-14. 10.2214/AJR.09.3224

9. Wattanasatesiri T, Puntu W, Vithitsuvanakul N: Influencing factors of pneumothorax and parenchymal haemorrhage after CT-guided transthoracic needle biopsy: single-institution experience. Pol J Radiol. 2018, 83:e379-88. 10.5114/pjr.2018.79202

10. Vagn-Hansen C, Pedersen MR, Rafaelsen SR: Diagnostic yield and complications of transthoracic computed tomography-guided biopsies. Dan Med J. 2016, 63:A5239.

11. Tuna T, Ozkaya S, Dirican A, Findik S, Atici AG, Erkan L: Diagnostic efficacy of computed tomographyguided transthoracic needle aspiration and biopsy in patients with pulmonary disease. Onco Targets Ther. 2013, 6:1553-7. 10.2147/OTT.S45013

12. Nour-Eldin NE, Alsubhi M, Emam A, et al.: Pneumothorax complicating coaxial and non-coaxial CT-guided lung biopsy: comparative analysis of determining risk factors and management of pneumothorax in a retrospective review of 650 patients. Cardiovasc Intervent Radiol. 2016, 39:261-70. 10.1007/s00270-0151167-3

13. Wang Y, Li W, He X, Li G, Xu L: Computed tomography-guided core needle biopsy of lung lesions: diagnostic yield and correlation between factors and complications. Oncol Lett. 2014, 7:288-94. 10.3892/ol.2013.1680

14. Cruz JF, Rolo R, Iglésias L, Cunha J: CT-guided transthoracic lung biopsy: predictive factors of pneumothorax. Rev Port Pneumol. 2014, 20:174-6. 10.1016/j.rppneu.2014.01.009

15. Niu XK, Bhetuwal A, Yang HF: CT-guided core needle biopsy of pleural lesions: evaluating diagnostic yield and associated complications. Korean J Radiol. 2015, 16:206-12. 10.3348/kjr.2015.16.1.206 